\title{
Lateral migration of flexible fibers in Poiseuille flow between two parallel planar solid walls
}

\author{
Agnieszka M. Słowicka, Eligiusz Wajnryb, and Maria L. Ekiel-Jeżewska ${ }^{a}$ \\ Institute of Fundamental Technological Research, Polish Academy of Sciences, Pawińskiego 5B, 02-106, Warsaw, Poland
}

Received 5 January 2012 and Received in final form 12 August 2012

Published online: 28 March 2013

(c) The Author(s) 2013. This article is published with open access at Springerlink.com

\begin{abstract}
Dynamics of non-Brownian flexible fibers in Poiseuille flow between two parallel planar solid walls is evaluated from the Stokes equations which are solved numerically by the multipole method. Fibers migrate towards a critical distance from the wall $z_{c}$, which depends significantly on the fiber length $N$ and bending stiffness $A$. This effect can be used to sort fibers. Three types of accumulation are found, depending on a shear-to-bending parameter $\Gamma$. In the first type, stiff fibers deform only a little and accumulate close to the wall, where their tendency to drift away from the channel is balanced by the repulsive hydrodynamic interaction with the wall. In the second type, flexible fibers deform significantly and accumulate far from the wall. In both types, the fiber shapes at the accumulation positions are repeatable, while in the third type, they are very compact and non-repeatable. The difference between the second and third accumulation types is a special case of the difference between the regular and irregular modes for the dynamics of migrating fibers. At the regular mode, far from walls, the fiber tumbling frequency satisfies Jeffery's expression, with the local shear rate and the aspect ratio close to $N$.
\end{abstract}

\section{Introduction}

Dynamics of flexible fibers in simple shear and Poiseuille flows has been analyzed theoretically, numerically and experimentally in numerous publications [1-12]. Migration of fibers or vesicles in Poiseuille flow [13-19] is the fundamental problem of modern lab-on-chip hydrodynamics, important in various biological, medical and industrial contexts, such as Brownian dynamics of proteins, actins, DNA or biological polymers, cell motion, swimming of microorganisms, drug delivery, transport of microparticles [20-22].

For significant pressure differences, corresponding to large maximal flow velocities, migration is caused by fluid inertia [23]. However, fluid flows in microchannel devices often take place at low-Reynolds-numbers. In such systems, Brownian rigid rods migrate towards the wall [13, 14], and flexible fibers to an off-center position [24-27].

For non-Brownian systems, the key question is under what conditions there exist off-center distances from microchannel walls where flexible fibers tend to accumulate, what are their values, and how they depend on the fiber size, aspect ratio and flexibility. The importance of this problem is straightforward. Focusing of micro and nanoparticles is essential for their counting, detecting, and sorting $[28,29]$.

\footnotetext{
a e-mail: mekiel@ippt.pan.pl
}

The dynamics of flexible fibers is also interesting from the fundamental point of view [30]. Evolution of their nonstraight shapes is related to the existence of a family of modes, which are activated if the characteristic parameter exceeds subsequent threshold values. The parameter is determined as the ratio of the viscous forces to the bending ones [9-12].

In this paper, we study both practical and fundamental aspects of the fiber dynamics. We investigate where the fibers accumulate, using the bead model and the multipole method [31] of solving the Stokes equations, implemented in a very accurate, well-tested HYDROMULTIPOLE numerical code [32]. The goal is to determine how position of accumulation planes depends on the the fiber bending stiffness and its length, and to relate the findings to the characteristic parameter and its thresholds. In sect. 2, we specify the system and theoretical model. The results are presented in sect. 3 . In sect. 4 we conclude, discussing different modes of the fiber dynamics and thresholds of the characteristic parameter.

\section{System}

\subsection{Fluid flow}

We analyze motion and shape deformation of a single nonBrownian flexible fiber, moving freely in Poiseuille flow 


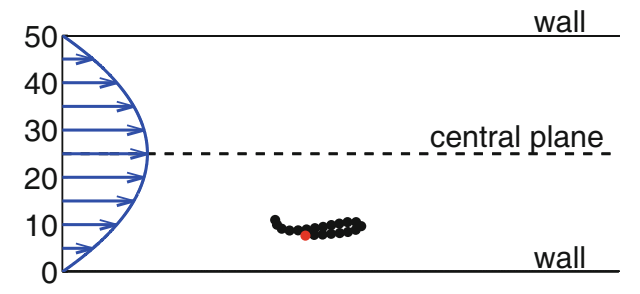

Fig. 1. A flexible fiber entrained by Poiseuille flow between two parallel solid walls.

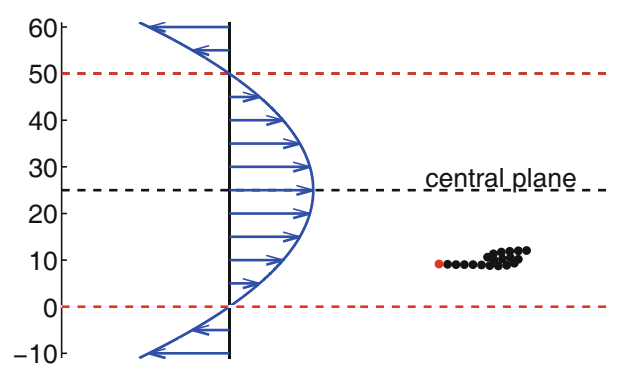

Fig. 2. Reference system: a flexible fiber entrained by unbounded Poiseuille flow (without walls).

inside a channel made of two parallel solid walls, as illustrated in fig. 1 . The fluid velocity $\mathbf{v}$ and pressure $p$ satisfy the stationary Stokes equations [33,34]

$$
\eta \boldsymbol{\nabla}^{2} \mathbf{v}-\nabla p=\mathbf{0} \quad \text { and } \quad \boldsymbol{\nabla} \cdot \mathbf{v}=0
$$

where $\eta$ is the fluid dynamic shear viscosity.

The fluid is confined between two parallel infinite solid walls at $z=0$ and $z=h$, with the Poiseuille flow velocity

$$
\mathbf{v}_{0}=4 z(h-z) / h^{2} \hat{\mathbf{x}} .
$$

The stick boundary conditions are satisfied at the surface of the fiber and at the solid walls, which confine the fluid. At infinity, the fluid velocity $\mathbf{v}=\mathbf{v}_{0}$.

Distances are normalized by the fiber thickness $d$, velocities by the maximal velocity $v_{m}$ of the Poiseuille flow, forces by $f_{0}=\pi \eta d v_{m}$, and time by $t_{0}=d / v_{m}$.

The system defined above is important for practical applications, but complex to be studied theoretically. First, the shear rate depends on the position $z$ across the channel, and second, the hydrodynamic interaction of the fiber with the walls is significant. To separate these two effects, we also study a reference system (see fig. 2), with the Poiseuille flow given by the same eq. (2), but not bounded by the walls, and extending beyond $0 \leq z \leq h$.

\subsection{Fiber dynamics}

A single fiber consists of $N$ solid spherical beads of diameter $d$ equal to the fiber thickness [35]. Owing to nonhydrodynamic constraints, the beads do not move apart. There are no non-hydrodynamic torques, and the nonhydrodynamic force exerted on each bead $i=1, \ldots, N$ by its neighbors is the sum of the elastic and bending forces $[20], \boldsymbol{F}_{i}=\boldsymbol{F}_{i}^{e}+\boldsymbol{F}_{i}^{b}$, with

$$
\begin{aligned}
\boldsymbol{F}_{i}^{e} & =-k\left(l_{i}-l_{0}\right) \hat{\boldsymbol{t}}_{i}+k\left(l_{l+1}-l_{0}\right) \hat{\boldsymbol{t}}_{i+1}, \\
\boldsymbol{F}_{i}^{b} & =-\frac{A}{2 l_{0}} \boldsymbol{\nabla}_{i} \sum_{n=2}^{N-1}\left(\hat{\boldsymbol{t}}_{n+1}-\hat{\boldsymbol{t}}_{n}\right)^{2},
\end{aligned}
$$

where $k$ is the ratio of Hooke's constant to $f_{0} / d$ and $A$ is the ratio of the bending stiffness to $f_{0} d^{2}$ (in the following just called the bending stiffness). In the above equation, $l_{0}$ and $l_{i}$ denote the equilibrium and time-dependent distances between the centers of the consecutive beads, respectively, with $l_{i}=\left|\boldsymbol{t}_{i}\right|$, where $\boldsymbol{t}_{i}=\boldsymbol{r}_{i}-\boldsymbol{r}_{i-1}$ is the difference between the positions $\boldsymbol{r}_{k}$ of the consecutive bead centers $k=i-1, i$. Here, $\hat{\boldsymbol{t}}_{i}=\boldsymbol{t}_{i} / l_{i}$ and $\boldsymbol{\nabla}_{i}$ is the derivative with respect to $\boldsymbol{r}_{i}$. The total non-hydrodynamic force applied to all the fiber beads vanishes, $\sum_{i=1}^{N} \boldsymbol{F}_{i}=0$.

Translational and rotational velocities of the fiber beads, $\mathbf{U}=\left(\mathbf{U}_{1}, \ldots, \mathbf{U}_{N}\right)$ and $\boldsymbol{\Omega}=\left(\boldsymbol{\Omega}_{1}, \ldots, \boldsymbol{\Omega}_{N}\right)$, are linear combinations of the non-hydrodynamic forces $\boldsymbol{F}=$ $\left(\boldsymbol{F}_{1}, \ldots, \boldsymbol{F}_{N}\right)$ exerted on them all, and the multipoles of the ambient velocity field (2), with the coefficients determined by the elements of the grand mobility matrix [36]. All the terms related to the ambient flow can be interpreted as resulting from the hydrodynamic forces $\boldsymbol{F}_{0}=$ $\left(\boldsymbol{F}_{01}, \ldots, \boldsymbol{F}_{0 N}\right)$ and torques $\boldsymbol{T}_{0}=\left(\boldsymbol{T}_{01}, \ldots, \boldsymbol{T}_{0 N}\right)$, exerted by the same ambient flow (2) on motionless beads fixed at the same instantaneous positions as the fiber beads. Therefore

$$
\left(\begin{array}{l}
\mathbf{U} \\
\boldsymbol{\Omega}
\end{array}\right)=\boldsymbol{\mu} \cdot\left(\begin{array}{c}
\boldsymbol{F}+\boldsymbol{F}_{0} \\
\boldsymbol{T}_{0}
\end{array}\right),
$$

with the mobility matrix $\boldsymbol{\mu}$ dependent on the instantaneous positions of all the bead centers, $\boldsymbol{r}=\left(\mathbf{r}_{1}, \ldots, \mathbf{r}_{N}\right)$.

For a given configuration, values of $\boldsymbol{F}_{0}, \boldsymbol{T}_{0}$ and $\boldsymbol{\mu}$ are determined by the multipole expansion of the Stokes equations [31,37], with the wall effects evaluated by the single-wall superposition $[38,39]$. The computations are performed with the use of the HYDROMULTIPOLE numerical code [32]. Then, the adaptive fourth-order RungeKutta method is applied to determine the fiber dynamics,

$$
\mathrm{d} \boldsymbol{r} / \mathrm{d} t=\mathbf{U} .
$$

Initially, the fiber is aligned with the flow (i.e. along the $x$ axis), with the bead centers located at

$$
\boldsymbol{r}_{i}(t=0)=\left(i l_{0}, 0, z_{0}\right), \quad \text { for } \quad i=1, \ldots, N .
$$

Owing to symmetry, the fiber moves in the $x z$ plane. The computations are three-dimensional, and no deformation of the fiber out of the plane is observed.

\subsection{Parameters}

In the numerical simulations, we have used single values of the dimensionless channel width $h$, Hooke's constant $k$ and equilibrium distance between the consecutive beads $l_{0}$,

$$
h=50, \quad k=80, \quad l_{0}=1.01 \text {. }
$$



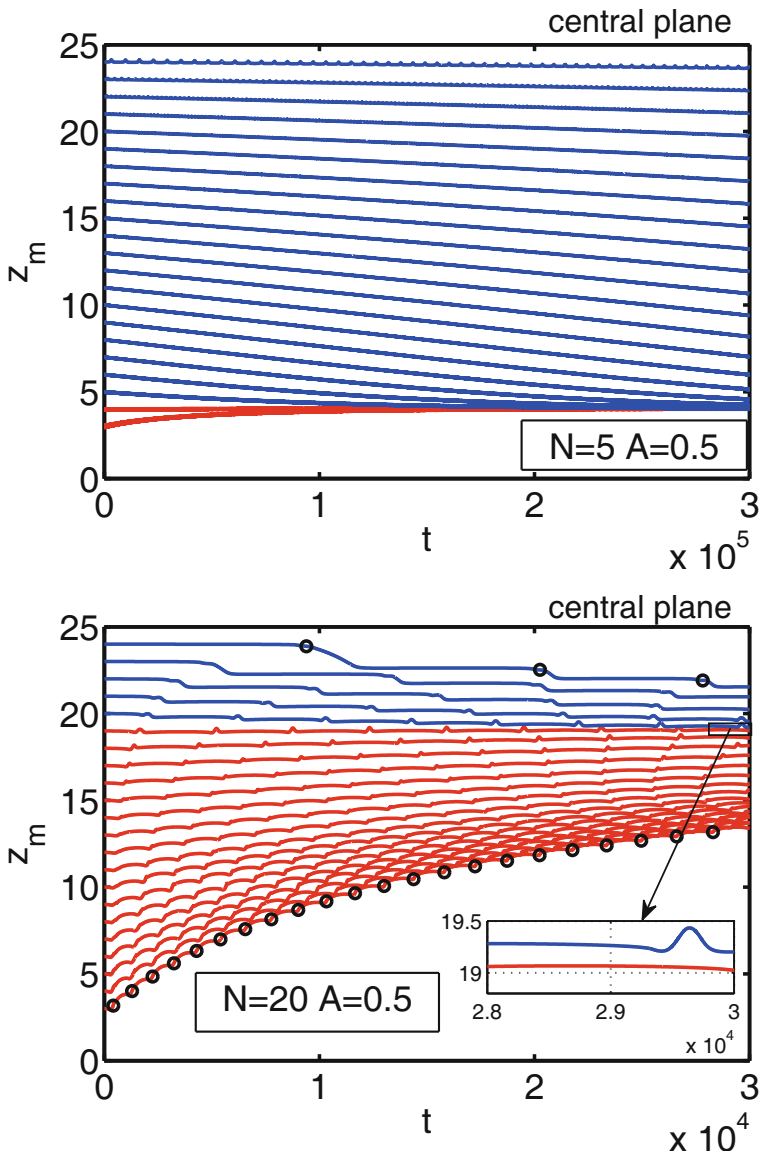
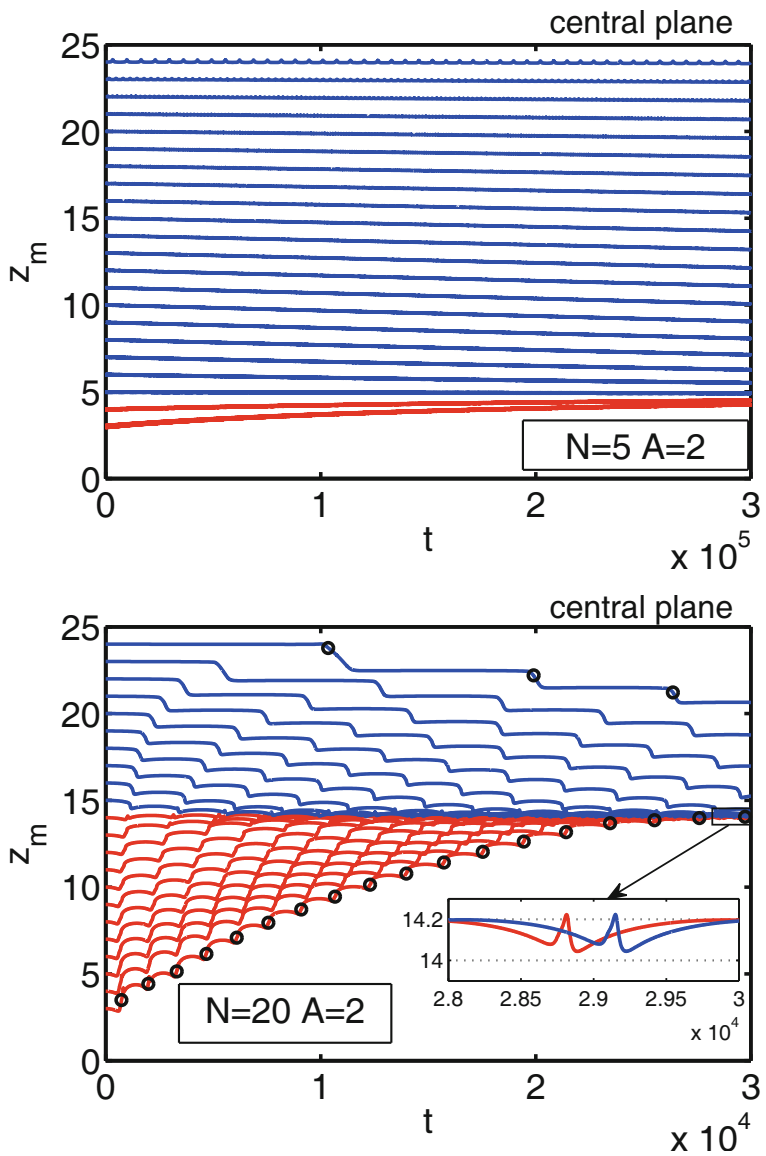

Fig. 3. Evolution of the distance $z_{m}(t)$ from the fiber center of mass to the wall, for fibers initially aligned with the flow. Here, $z=0$ and $z=25$ correspond to the wall and the central plane of the channel, respectively. Black circles denote flipping instants.

Table 1. The fiber length $L=N / h$ as a fraction of the channel width for the fiber aspect ratio $N$ used in the simulations.

\begin{tabular}{cccc}
\hline$N$ & 5 & 10 & 20 \\
\hline$L$ & 0.1 & 0.2 & 0.4 \\
\hline
\end{tabular}

A large value of $k$ and small gap size $\left(l_{0}-1\right)$ between the beads are chosen to model compact fibers which practically do not change their length while bending.

Three different numbers of beads $N$ have been considered, with the corresponding fiber aspect ratio (or length), $(N-1) l_{0}+1$, practically equal to $N$. The corresponding fraction of the channel width, $L=N / h$, is explicitly given in table 1. For clarity of presentation, we focus on discussing in details the results obtained for $N=10$.

Computations have been performed for a wide range of the initial fiber positions $z_{0}$ across the channel. The values of the bending stiffness $A$ ranged from $0.01 \leq A \leq 4$, and have been chosen to observe thresholds for different types of the accumulation and different modes of the dynamics. It is known [9-12] that the transitions between C, S and $\mathrm{W}$ modes (with the names referring to the corresponding fiber shapes) are associated with critical values of a dimensionless parameter, equal to the ratio of the viscous forces (proportional to the local shear rate) to the bend- ing ones. This parameter is widely used to characterize systems, which are far from interfaces. However, it is clear that under confinement (as in the system considered in this work), there are additional wall effects which influence thresholds of the fiber dynamics. In this paper, we study these effects, by comparing the systems with (fig. 1) and without (fig. 2) walls. We use two basic parameters $N$ and $A$ to describe the fiber evolution. For $N=10$, and given values of $A$ and the distance $z_{m}$ from the fiber center-of-mass to the closer wall, we evaluate a shear-tobending dimensionless number mentioned above,

$$
\Gamma=\left(h / 2-z_{m}\right) / A,
$$

and find out its critical values. We remind that $h, z_{m}$ and $A$ are dimensionless (normalized by $d$ and $\pi \eta d^{3} v_{m}$, respectively).

\section{Results}

\subsection{Lateral migration and accumulation positions}

In ref. [40], dynamics of fibers in the same system was analyzed, focusing on the migration towards the central plane of the channel and its dependence on the fiber stiffness, 
Table 2. The distance $z_{c}$ from the wall where fibers accumulate while flipping inside the channel bounded by two walls.

\begin{tabular}{|c|c|c|c|c|c|c|c|c|c|c|c|c|c|}
\hline$N \backslash A$ & 0.025 & 0.04 & 0.05 & 0.06 & 0.125 & 0.2 & 0.25 & 0.38 & 0.46 & 0.5 & 1.0 & 2.0 & 4.0 \\
\hline 5 & $\begin{array}{l}16.81 \\
( \pm 0.05) \\
3.6 \\
( \pm 0.1)\end{array}$ & $\begin{array}{l}12.06 \\
( \pm 0.01)\end{array}$ & $\begin{array}{l}9.11 \\
( \pm 0.05)\end{array}$ & $\begin{array}{l}6.58 \\
( \pm 0.01)\end{array}$ & $\begin{array}{l}4.48 \\
( \pm 0.02)\end{array}$ & $\begin{array}{l}4.3 \\
( \pm 0.1)\end{array}$ & $\begin{array}{l}4.19 \\
( \pm 0.02)\end{array}$ & $\begin{array}{l}.07 \\
( \pm 0.1)\end{array}$ & $\begin{array}{l}.03 \\
( \pm 0.1)\end{array}$ & $\begin{array}{l}4.02 \\
( \pm 0.05)\end{array}$ & $\begin{array}{l}4.3 \\
( \pm 0.1)\end{array}$ & $\begin{array}{l}4.7 \\
( \pm 0.1)\end{array}$ & \\
\hline 10 & & $\begin{array}{l}22.79 \\
( \pm 0.05) \\
11.4 \\
( \pm 0.4)\end{array}$ & $\begin{array}{l}22.26 \\
( \pm 0.05) \\
10.2 \\
( \pm 0.7)\end{array}$ & $\begin{array}{l}21.79 \\
( \pm 0.05) \\
8.0 \\
( \pm 0.5)\end{array}$ & $\begin{array}{l}17.97 \\
( \pm 0.03)\end{array}$ & $\begin{array}{l}14.47 \\
( \pm 0.02)\end{array}$ & $\begin{array}{l}12.37 \\
( \pm 0.02)\end{array}$ & $\begin{array}{l}8.80 \\
( \pm 0.05)\end{array}$ & $\begin{array}{l}8.25 \\
( \pm 0.05)\end{array}$ & $\begin{array}{l}8.20 \\
( \pm 0.05)\end{array}$ & $\begin{array}{l}7.5 \\
( \pm 0.1)\end{array}$ & $\begin{array}{l}6.4 \\
( \pm 0.1)\end{array}$ & \\
\hline 20 & & & & & & & $\begin{array}{l}21.28 \\
( \pm 0.1)\end{array}$ & $\begin{array}{l}20.1 \\
( \pm 0.1)\end{array}$ & $\begin{array}{l}19.4 \\
( \pm 0.1)\end{array}$ & $\begin{array}{l}19.1 \\
( \pm 0.2)\end{array}$ & $\begin{array}{l}15.9 \\
( \pm 0.1)\end{array}$ & $\begin{array}{l}14.2 \\
( \pm 0.1)\end{array}$ & $\begin{array}{l}13.4 \\
( \pm 0.1)\end{array}$ \\
\hline
\end{tabular}

aspect ratio and distance from the wall. But for certain values of these parameters, fibers migrate away from the central plane. In this paper, we determine the critical distance $z_{c}$ from the wall where fibers accumulate.

Figure 3 shows evolution of the distance $z_{m}(t)$ between the fiber center of mass and the closer wall, starting from different values $z_{m}(0)=z_{0}$. Online, positions of the fibers, which move towards (away from) the central plane of the channel are plotted in red (blue).

All fibers tend to an off-center position across the flow. For $N=5$, the migration rate is significantly slower than for $N=20$ (notice the 10 times larger range of times of the upper plots in fig. 3 ).

The lateral migration of fibers is superimposed with oscillations of their center-of-mass position, clearly visible in fig. 3 for $N=20$. These oscillations are related to the fiber tumbling motion, caused by the local shear of the flow. A flipping time $t_{f}$ is defined as the instant when the end-to-end vector (which links the centers of the first and the last beads of the fiber) becomes perpendicular to the flow direction. The distance from the fiber center-of-mass to the wall at time $t_{f}$ will be denoted as $z_{f}$

$$
z_{f} \equiv z_{m}\left(t_{f}\right)
$$

In fig. 3 , consecutive positions $z_{f}$ are marked at four selected simulation runs with $N=20$. It is clear that

$$
z_{f} \longrightarrow z_{c} \quad \text { when } \quad t \longrightarrow \infty \text {. }
$$

In the following, we will call $z_{c}$ "the accumulation position" or "the accumulation distance". Some of the evaluated values of $z_{c}$ are listed in table 2 . Their relative accuracy (typically, 0.5-2\%), is determined as the maximum of the oscillation amplitude and the half-a-distance between the closest decreasing and increasing curves $z_{m}\left(t_{f}\right)$, at the last flipping instant $t_{f}$ observed in our simulations.

For $N=10$, the results are shown in fig. 4. Stiff fibers (circles, blue online) accumulate close to the wall, at $N / 2<z_{c}<N$, and $z_{c}$ only slightly increases with the decrease of $A$. However, for flexible fibers (squares, green online), $z_{c}$ rapidly increases with the decreasing $A$. For a sufficiently small value of $A$, a second accumulation plane is also observed (diamond, violet purple online).

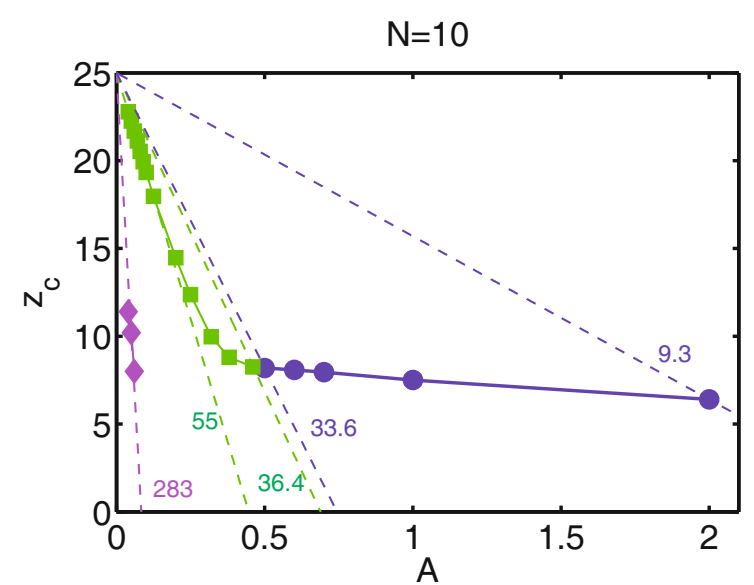

Fig. 4. The distance $z_{c}$ from the wall where flipping fibers accumulate, versus their bending stiffness $A$. Dashed lines indicate constant values of $\Gamma=\left(h / 2-z_{c}\right) / A$.

\subsection{Comparison with unbounded Poiseuille flow helps to discriminate between two accumulation mechanisms}

It seems that accumulation of stiff fibers close to the channel boundary is caused by their interaction with the wall, which prevents them from escaping. Accumulation of flexible fibers far from the wall, however, seems to be caused by another mechanism - their shape deformation and the flow curvature. This hypothesis will be now verified.

In table 3 , we evaluate accumulation positions $z_{c}^{\text {no-wall }}$ for the same fibers and the same ambient flow as in the previous section, but without walls (the system shown in fig. 2). Comparison with table 2 shows that more flexible fibers accumulate at similar positions with and without the walls, $z_{c} \sim z_{c}^{\text {no-wall }}$. An example is illustrated in the left panel of fig. $5(N=10$ and $A=0.2)$. However, the motion of more stiff fibers significantly depends on the presence or absence of the walls. The difference can be seen by comparing the top and bottom plots in the right panel of fig. $5(N=10$ and $A=2)$. With walls, the fibers accumulate at $z_{f}=z_{c}$ inside the channel. Without walls, the fibers migrate out of the "channel regime" (defined as 
Table 3. The position $z_{c}^{\text {no-wall }}$ where flipping fibers accumulate in the absence of walls. The arrows $\searrow$ indicate that there is no accumulation position - all fibers migrate away towards $z_{m}<0$.

\begin{tabular}{|c|c|c|c|c|c|c|c|c|c|c|}
\hline$N \backslash A$ & 0.025 & 0.05 & 0.125 & 0.2 & 0.25 & 0.38 & 0.46 & 0.5 & 2.0 & 4.0 \\
\hline 5 & $\begin{array}{l}16.8 \\
( \pm 0.1)\end{array}$ & $\begin{array}{l}8.65 \\
( \pm 0.05)\end{array}$ & $\searrow$ & $\searrow$ & $\searrow$ & $\searrow$ & $\searrow$ & $\searrow$ & $\searrow$ & \\
\hline 10 & & $\begin{array}{l}22.2 \\
( \pm 0.05) \\
5.8 \\
( \pm 0.1)\end{array}$ & $\begin{array}{l}17.85 \\
( \pm 0.05)\end{array}$ & $\begin{array}{l}14.2 \\
( \pm 0.1)\end{array}$ & $\begin{array}{l}11.76 \\
( \pm 0.02)\end{array}$ & $\begin{array}{l}5.49 \\
( \pm 0.05)\end{array}$ & $\begin{array}{l}1.4 \\
( \pm 0.1)\end{array}$ & $\searrow$ & $\searrow$ & \\
\hline 20 & & & & & $\begin{array}{l}21.4 \\
( \pm 0.2)\end{array}$ & $\begin{array}{l}20.0 \\
( \pm 0.15)\end{array}$ & $\begin{array}{l}19.1 \\
( \pm 0.1)\end{array}$ & $\begin{array}{l}18.6 \\
( \pm 0.1)\end{array}$ & $\begin{array}{l}2.6 \\
( \pm 0.1)\end{array}$ & $\searrow$ \\
\hline
\end{tabular}

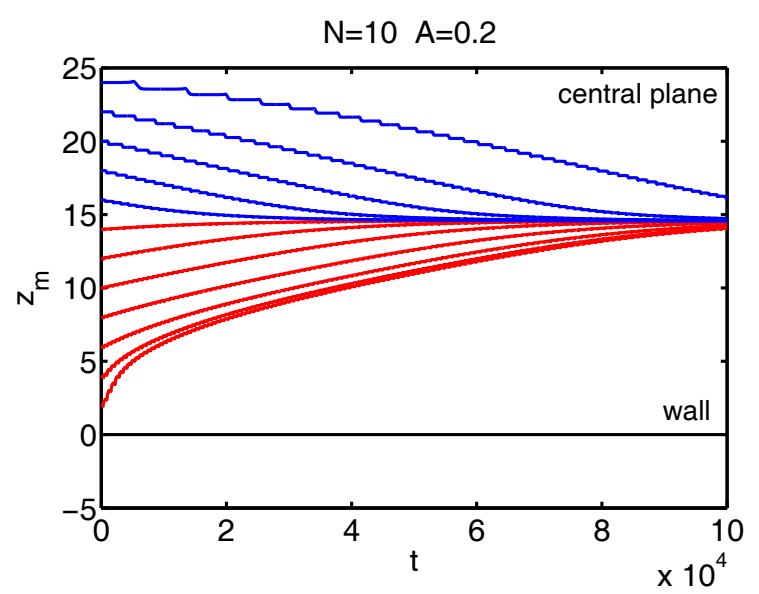

$\mathrm{N}=10 \quad \mathrm{~A}=0.2$

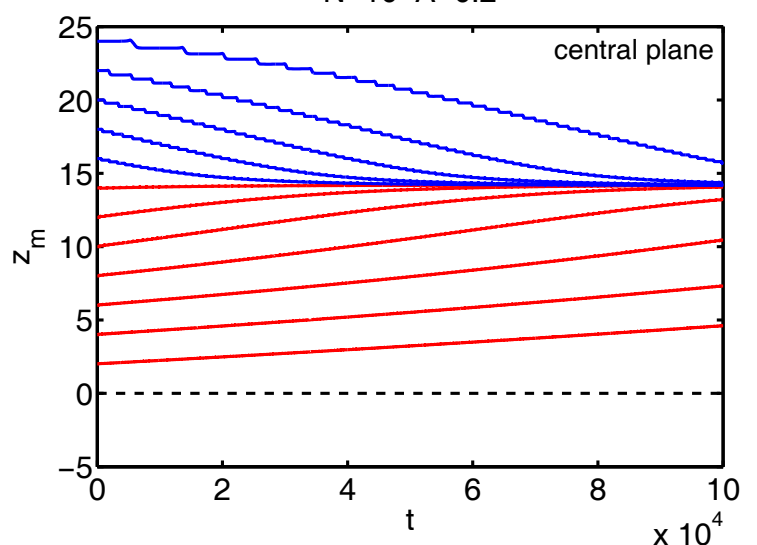

$\mathrm{N}=10 \quad \mathrm{~A}=2$

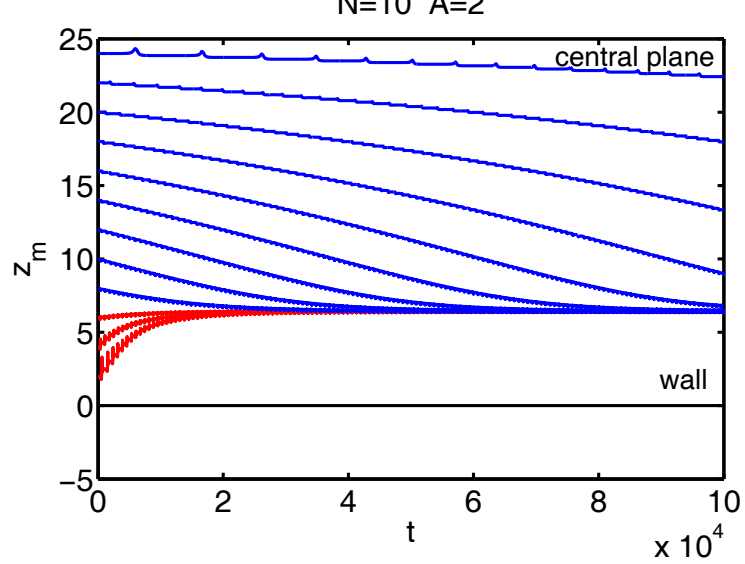

$\mathrm{N}=10 \quad \mathrm{~A}=2$

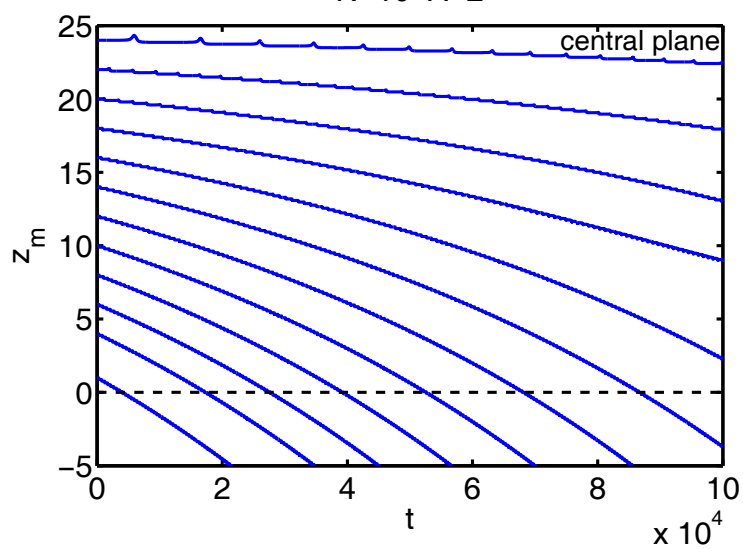

Fig. 5. Evolution of the distance $z_{m}(t)$ from a fiber center of mass to the closer plane, where the ambient Poiseuille flow vanishes. Top: the flow bounded by the walls, as in fig. 1. Bottom: the same fiber and the flow, but without walls, as in fig. 2 .

$\left.0 \leq z_{f} \leq h\right)$, whatever is their initial position across the flow; there is no accumulation points in this range of $z_{f}$. In table 3 , such a behavior is indicated by arrows pointing down-right.

Clearly, there exist two mechanisms of fiber accumulation inside the channel. The first type of the fiber accumulation is caused by its hydrodynamic interaction with the wall (blue circles in fig. $4, A \geq 0.50, \Gamma \leq 33.6$ ). The second type of the fiber accumulation is caused by its interaction with the flow itself (green squares in fig. $4, A \leq 0.46$,
$\Gamma \geq 36.4$ ). The parameter $\Gamma$ is defined by eq. (9), with the center-of-mass taken at the flipping instant, $z_{m} \equiv z_{f}$, and moreover, at the accumulation position, $z_{f}=z_{c}$. The transition between these two accumulation types takes place for critical values of $A_{0}$, or equivalently, $\Gamma_{0}$, such that

$$
\begin{aligned}
& 0.50 \geq A_{0} \geq 0.46 \\
& 33.6 \leq \Gamma_{0} \leq 36.4 .
\end{aligned}
$$




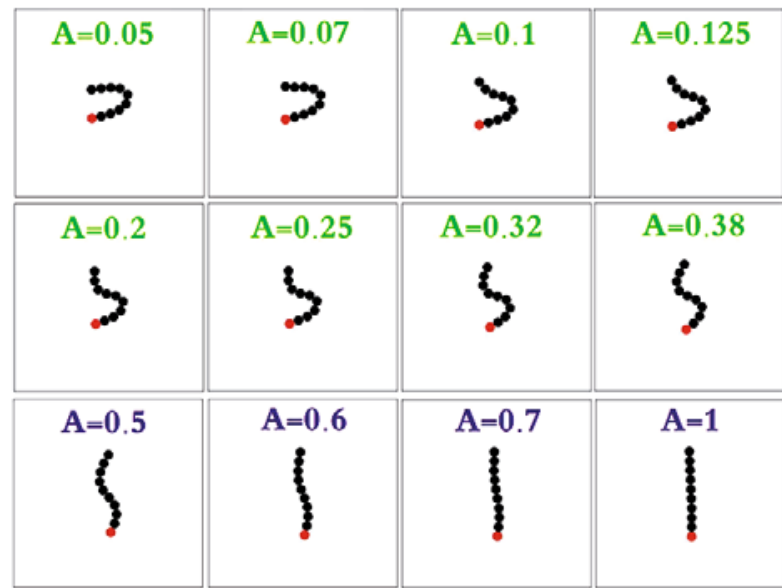

Fig. 6. Fiber shapes at the accumulation positions $z_{c}$ of the first and second types, for a different bending stiffness $A$.

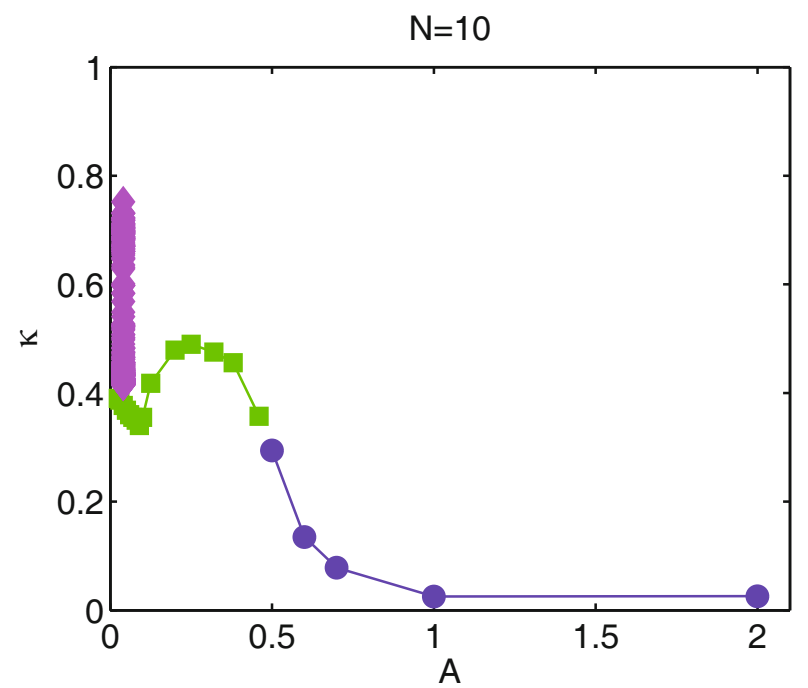

Fig. 7. The fiber curvature $\kappa$ at the accumulation position $z_{c}$, versus its bending stiffness $A$.

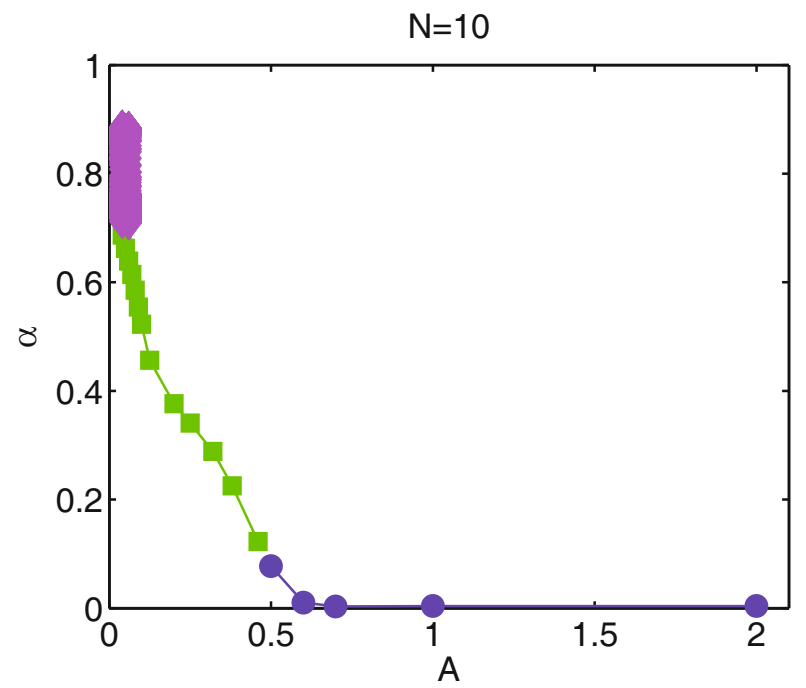

Fig. 8. The fiber fractional compression $\alpha$ at the accumulation position, versus its bending stiffness $A$.

\subsection{Fiber shapes at the accumulation positions $z_{c}$}

We will now investigate if different accumulation types are correlated with different fiber shapes at the accumulation positions $z_{c}$.

In fig. 6 , the fiber shapes at the accumulation positions $z_{c}$ are shown for different values of the bending stiffness $A$. The bottom row corresponds to the first accumulation type (blue online), and the top and middle rows to the second accumulation type (green online). Clearly, for the first accumulation type, the fibers are less bent than for the second one. This stretching effect is caused by the hydrodynamic interaction of the fiber with the wall near by.

To quantify the difference of shapes, we introduce two parameters: the fiber curvature $\kappa$ (as in ref. [11]) and its fractional compression $\alpha$ (as in ref. [12]). The timedependent fiber curvature,

$$
\kappa=\frac{1}{N-2} \sum_{i=2}^{N-1} \frac{1}{R_{i}},
$$

is the mean inverse radius $1 / R_{i}$ of the circle determined by the centers of three consecutive beads.

The fiber fractional compression [12] is defined as

$$
\alpha=1-\delta\left(t_{f}\right) /(N-1),
$$

where $\delta\left(t_{f}\right)$ is the end-to-end distance of a fiber (i.e. the distance between the centers of its first and last beads) at the flipping time $t_{f}$.

In figs. 7 and 8 , we present values of $\kappa$ and $\alpha$ for fibers made of $N=10$ beads, located at the accumulation position $z_{c}$ at the flipping instants $t_{f}$.

From figs. 7 and 8 , it is evident that for the first accumulation type ( $A \geq 0.5$, blue circles), both the curvature and the fractional compression of the fibers flipping at the accumulation distance $z_{c}$ are smaller than for the second one ( $A \leq 0.46$, green squares), in agreement with the previous analysis of shapes at the accumulation positions, shown in fig. 6.

Comparing figs. 7 and 8 with fig. 6 , we conclude that the fiber curvature is very sensitive to a qualitative change of its shape, while the fractional compression is not sensitive at all. Indeed, with the decrease of $A, \alpha$ increases monotonically in the whole range of values, while $\kappa$ first increases from almost zero up to a local maximum (which is attained at $A$ around 0.25), next it falls down a bit, reaching a local minimum (around $A=0.09$ ), and then it increases again. These local extrema are correlated with the qualitative change of the fiber shapes; at the maximum, from the S-shaped to the $\varsigma$-shaped, and at the minimum, from $\varsigma$-shaped to C-shaped. The critical value $A_{0}$, which separates the first accumulation type from the second one, does not coincide with none of these extrema. This complex behavior results from the combined effects of the wall, flow curvature and the fiber flexibility.

Until now, only two types of the fiber accumulation have been discussed. As shown in fig. 4, for a very small 


\begin{tabular}{|c|c|c|c|}
\hline$A=0.05$ & $A=0.05$ & $A=0.05$ & $A=0.05$ \\
\hline & & & \\
\hline
\end{tabular}

Fig. 9. Fiber shapes at the accumulation positions $z_{c}$ of the third type, for $A=0.05$ and different flipping times $t_{f}$.

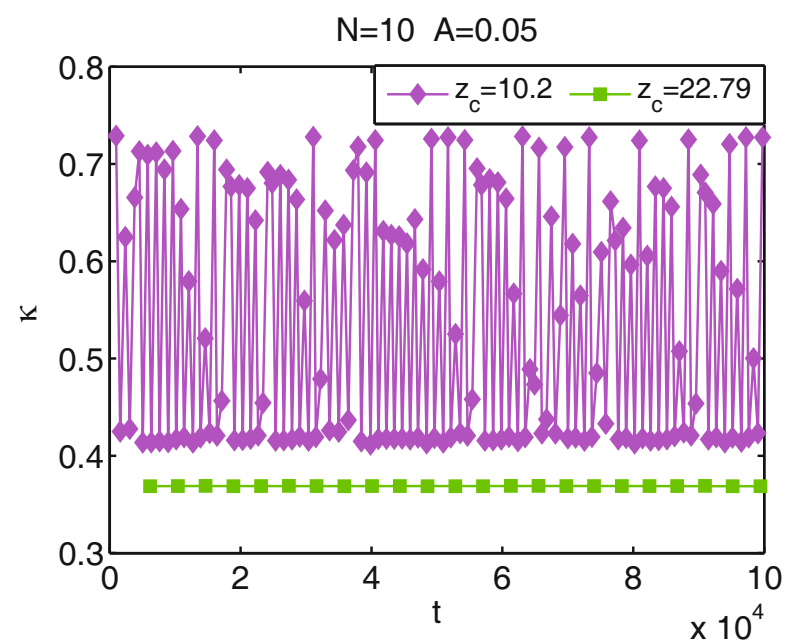

Fig. 10. The fiber curvature at the consecutive flipping instants, for the accumulation positions $z_{c}$ of the second (squares, green online) and third (diamonds, violet purple online) types.

value of the bending stiffness $A$ there appear two accumulation positions. We have not yet discussed this one which is closer to the wall (diamond, violet purple online in fig. 4), and belongs to a third type of the accumulation. This accumulation position (diamond, violet purple online in figs. 7 and 8) corresponds to a much higher curvature and fractional compression than the values observed for the first and second accumulation types.

As illustrated in fig. 9, at the accumulation positions $z_{c}$ of the third type, the fiber shapes are very compact; significantly more bent than the fiber shapes for the first and second accumulation types, shown in fig. 6 .

The essential feature of the third type of the accumulation is that for the same value of the bending stiffness $A$ and the same accumulation position $z_{c}$, but at different flipping instants, very different fiber shapes are observed, which are characterized by different, random values of the curvature and fractional compression. This irregular behavior occurs only for the third accumulation type; for the other ones, a given value of $A$ uniquely determines both $z_{c}$ and the fiber shape at $z_{c}$, for arbitrary flipping instants $t_{f}$.

Figure 10 illustrates the difference between the regular second accumulation type and the irregular third accumulation type. For $A=0.05$, there appear two accumulation positions $z_{c}$ : of the second and third types. In fig. 10, the fiber curvature at both these accumulation positions $z_{c}$ is plotted for a large number of the consecutive flipping instants $t_{f}$. For the accumulation position of the second type (squares, green online), $\kappa$ is the same for all $t_{f}$. However, for the accumulation position of the third type (diamonds, violet purple online), $\kappa$ changes irregularly from one flipping instant to the next one.

In fig. 11, we compare evolution of fiber shapes for times close to the flipping instants at the accumulation positions $z_{c}$ of the second and third types. The snapshots are labeled by the corresponding values of the rescaled time, $\bar{t} / \tau$, defined by the relations

$$
\begin{aligned}
& \bar{t}=t-t_{f}(2), \\
& \tau=t_{f}(3)-t_{f}(2),
\end{aligned}
$$

where $t_{f}(n)$ is the instant of the $n$-th flip. Therefore, $\bar{t}=0$ and $\bar{t}=1$ correspond to the second and third flipping instants, respectively.

At the trajectories of the second type (top and middle panels in fig. 11), fibers bend in a repeatable way, changing pattern almost periodically, with the half-period determined by the tumbling time $\tau$, which is the same between all the consecutive flips. In contrast, the shapes of fibers at the third mode (bottom panel) are not repeatable. In general, even the tumbling time between different pairs of the consecutive flips changes irregularly.

\subsection{Regular and irregular modes of the fiber dynamics}

Until now, we have focused on the fiber accumulation at the distance $z_{c}$ from the wall. It is also of interest to consider arbitrary positions $z_{m}$ of the fiber center of mass, and analyze general features of the fiber dynamics. The fiber shapes shown in fig. 11 indicate that there exist regular and irregular modes not only of the accumulation, but also of the entire fiber dynamics.

The essential features of the irregular mode are the irregular time dependence of the fiber center-of-mass position $z_{m}$, or a non-monotonic time dependence of $z_{f}$, related to a rapid variation of the tumbling time. In fig. 12, such irregular fluctuations are visible at the lower trajectories $z_{m}(t)$, which approach the accumulation distance $z_{c}=10.2$, in contrast to the regular oscillations of the fiber center of mass at the upper trajectories, which tend to $z_{c}=22.26$. Rapid changes of consecutive tumbling times appear at the bottom part of the lowest trajectory.

The properties described above can be used to determine such a critical value $\Gamma_{1}$ of the parameter $\Gamma=$ $\left(h / 2-z_{m}\right) / A$, which corresponds to the transition between the irregular $\left(\Gamma>\Gamma_{1}\right)$ and regular $\left(\Gamma<\Gamma_{1}\right)$ modes,

$$
259<\Gamma_{1}<275 \text {. }
$$

In fig. 13, we compare the time dependence of the fiber curvature for the regular and irregular modes. The reduced time $\bar{t} / \tau$ is defined by eqs. (16), (17). The regular mode (dashed line, green online) and the irregular mode with a lower curvature (solid line, violet purple online) correspond to the same two examples of the fiber dynamics, which are shown in the top and bottom panels of fig. 11, respectively. In both examples, the fiber center of mass is 


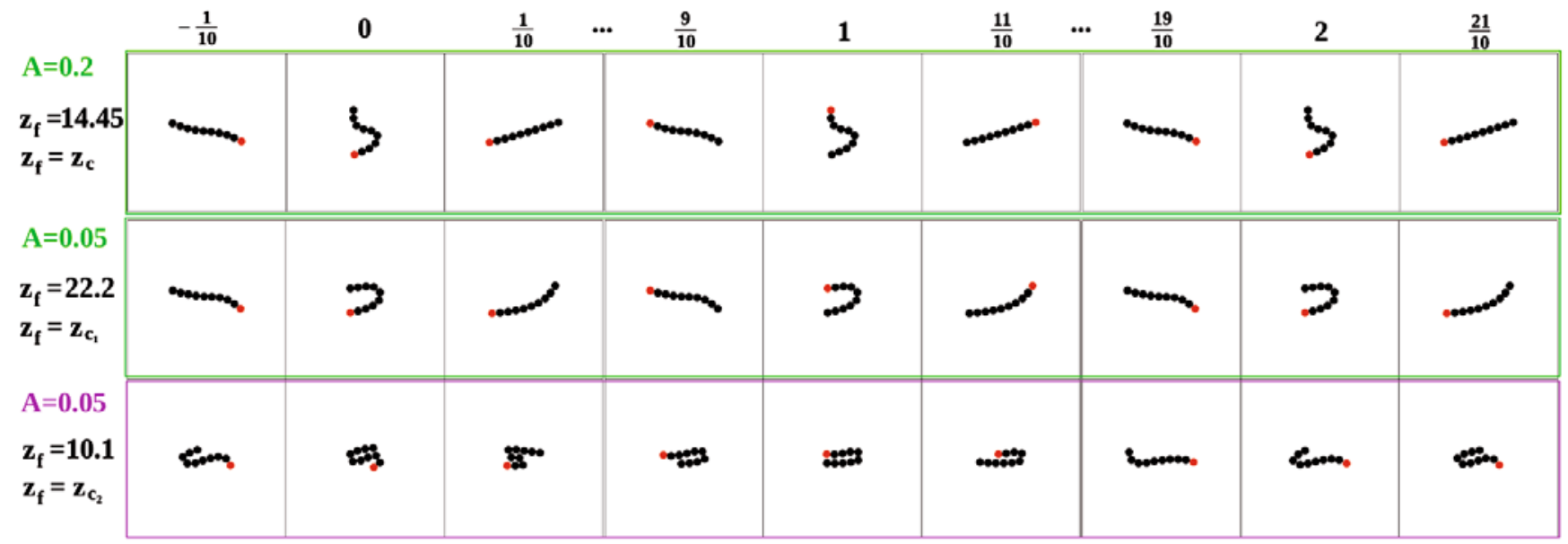

Fig. 11. Regular and irregular evolution of fibers with $N=10$, flipping around the accumulation positions $z_{c}$ of the second and third types, respectively. Snapshots from simulations are taken at the indicated values of time $\bar{t} / \tau$.

close to its accumulation position $z_{c}$, with $\Gamma \approx 53$ and $\Gamma \approx 296$, respectively. The fiber curvature $\kappa$ is periodic and smaller for the regular mode, and quasi-periodic and larger for the irregular one.

A third example is shown in fig. 13 as the dasheddotted curve (violet purple online), which corresponds to $\Gamma \approx 900$. The larger value of $\Gamma$ is related to the larger fiber curvature, and more irregular $z_{m}(t)$.

In the last part of this section, we focus on the regular mode, and investigate the corresponding fiber shapes at different positions $z_{m}$ and for different values of the bending stiffness $A$. In fig. 14, we compare evolution of flexible $(A=0.2)$ and stiff $(A=1)$ fibers in vicinity of the flipping instant, at the accumulation plane and also a bit out of it.

Figure 14 suggests that for stiff fibers, deformation is smaller than for flexible ones. At approximately the same distance from the wall, more flexible fibers are more curved than the stiff ones (compare the third and fourth rows). When accumulating close to the wall and interacting hydrodynamically with it, very stiff fibers are so stretched at the flipping moment, that their curvature at $z_{c}$ is close to zero.

Other features of the shape evolution seem to be very complex. According to fig. 13, during a single tumbling, the fiber curvature is often the largest at the flipping instant. But not always. In particular, stiff fibers at $z_{c}$ are almost straight, but they are bent before and after the flipping instant, see the fifth row.

In fig. 14, qualitatively different fiber shapes are observed - straight, C-shaped, s-shaped, and S-shaped. We compare shapes for different positions $z_{f}$ and different values of $A$. The corresponding values of the parameter $\Gamma$ are listed in fig. 14. For smaller values of $\Gamma$, the $\mathrm{C}$-shaped fibers are observed (rows 1, 4, 5 and 6), for larger ones, the $\varsigma$-shaped (row 2), and for even larger ones, the S-shaped (row 3). Such a transition to shape instability, triggered by a critical value of the shear-to-bending number (equivalent to a certain value of our parameter $\Gamma$ ) is known in the literature, see $[9,12]$ and the references within.

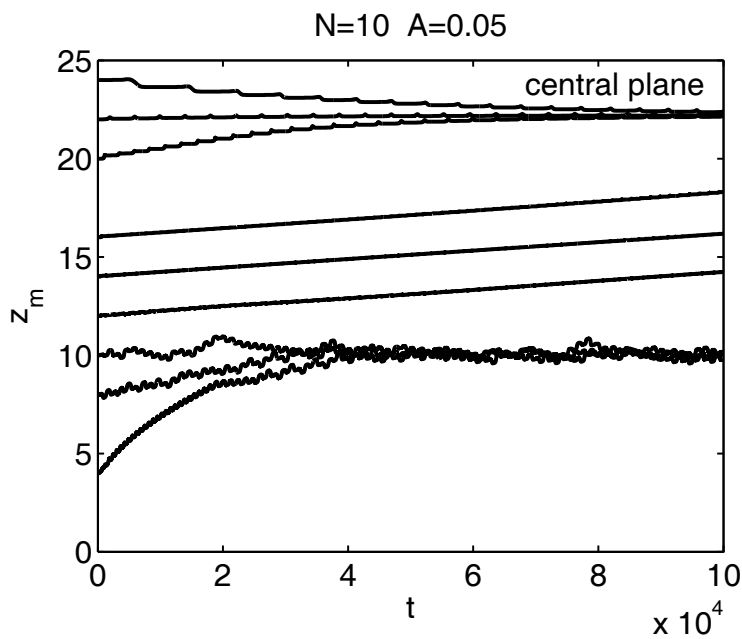

Fig. 12. Regular (top) and irregular (bottom) evolution of the distance from the fiber center of mass to the wall, $z_{m}(t)$.

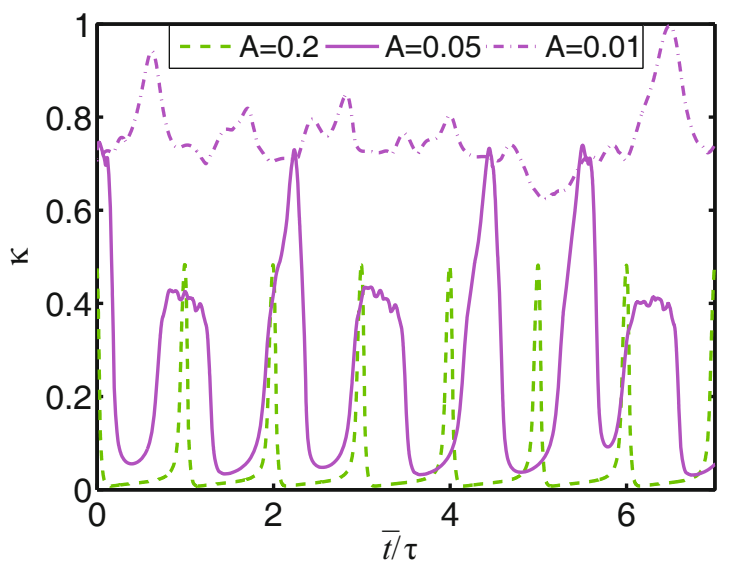

Fig. 13. The time-dependent curvature $\kappa$ of fibers with $N=10$ moving around the following positions $z_{m}$. Dashed line (green online): $z_{m} \approx z_{c}=14.47$. Solid line (violet purple online): $z_{m} \approx z_{c}=10.2$. Dashed-dotted line (violet purple online): $z_{m} \approx 16$. 

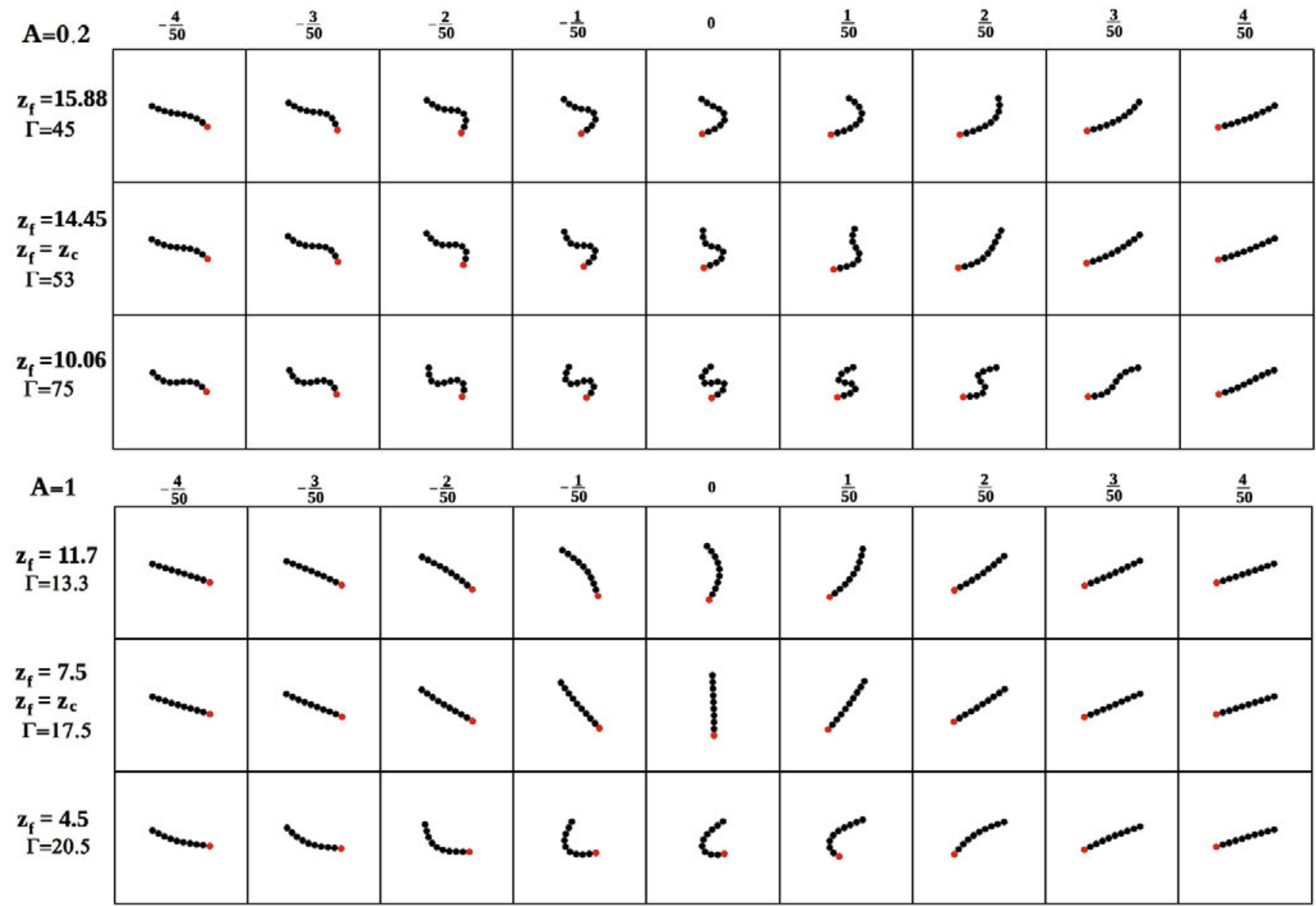

Fig. 14. Evolution of a fiber shape (drawn to scale) for $N=10$. Top: $A=0.2$. Bottom: $A=1$. Snapshots from simulations taken at the indicated times $\bar{t} / \tau$ (with $\bar{t}=t-t_{f}(2)$ and $\tau=t_{f}(3)-t_{f}(2)$, where $t_{f}(n)$ is the instant of the $n$-th flip). The indicated values of $z_{f}$ and $\Gamma$ are attained at $\bar{t}=0$ (second flip).

However, at the present stage, we cannot make any conclusive statements about a universal correlation between values of $\Gamma$ and specific shape modes in our system. For example, notice that the fiber evolution shown in the top panel of fig. 11 , with $\Gamma \approx 53$, is S-shaped, but in the middle panel, with the larger value $\Gamma \approx 56$, it is $\mathrm{C}$-shaped. This example indicates that the correlation between the value of $\Gamma$ and the shape mode is limited to some values of the system parameters. Its absence in fig. 11 might be related to the value of $z_{c}=22.26$ very close to the middle plane of the channel, or other reasons. Also, we should remember that in our system, there is a significant effect of the walls on the fiber dynamics, what interferes with the universal meaning of the parameter $\Gamma$, found for the unbounded systems $[9,12]$.

\subsection{Tumbling time}

It is interesting to determine how tumbling of fibers depends on their position across the channel. In fig. 15, the fiber flipping frequency $1 / \tau$ is plotted as a function of the distance $z_{f}$ from the wall at the flipping instant. For the regular mode, it is a monotonically decreasing function (except fibers, which are very close to the wall). For a wide range of larger distances from the wall, $1 / \tau$ is a linear function of $z_{f}$. Fibers at a larger distance from the wall tumble at a slower rate. Short fibers tumble more frequently in comparison to long fibers (notice a different range on vertical axis of each panel in fig. 15). Both effects are significant. The tumbling frequency is a bit larger for a smaller bending stiffness $A$. The difference is more pronounced for longer fibers.

Following the idea of Bretherton [41], widely used in various contexts $[1,2]$, we now compare the tumbling time $\tau$ characteristic for our flexible fibers entrained by the Poiseuille flow between two walls with the classic result of Jeffery for the rotation half-period $T / 2$ of rigid ellipsoids of revolution immersed in a simple shear flow in an unbounded fluid [42]. Jeffery derived the following relation between the rotation frequency $2 / T$ and the shear rate $\dot{\gamma}$,

$$
\frac{2}{T}=\frac{\dot{\gamma}}{\pi\left(r_{e}+1 / r_{e}\right)},
$$

where $r_{e}$ is the aspect ratio for the ellipsoid of revolution. 

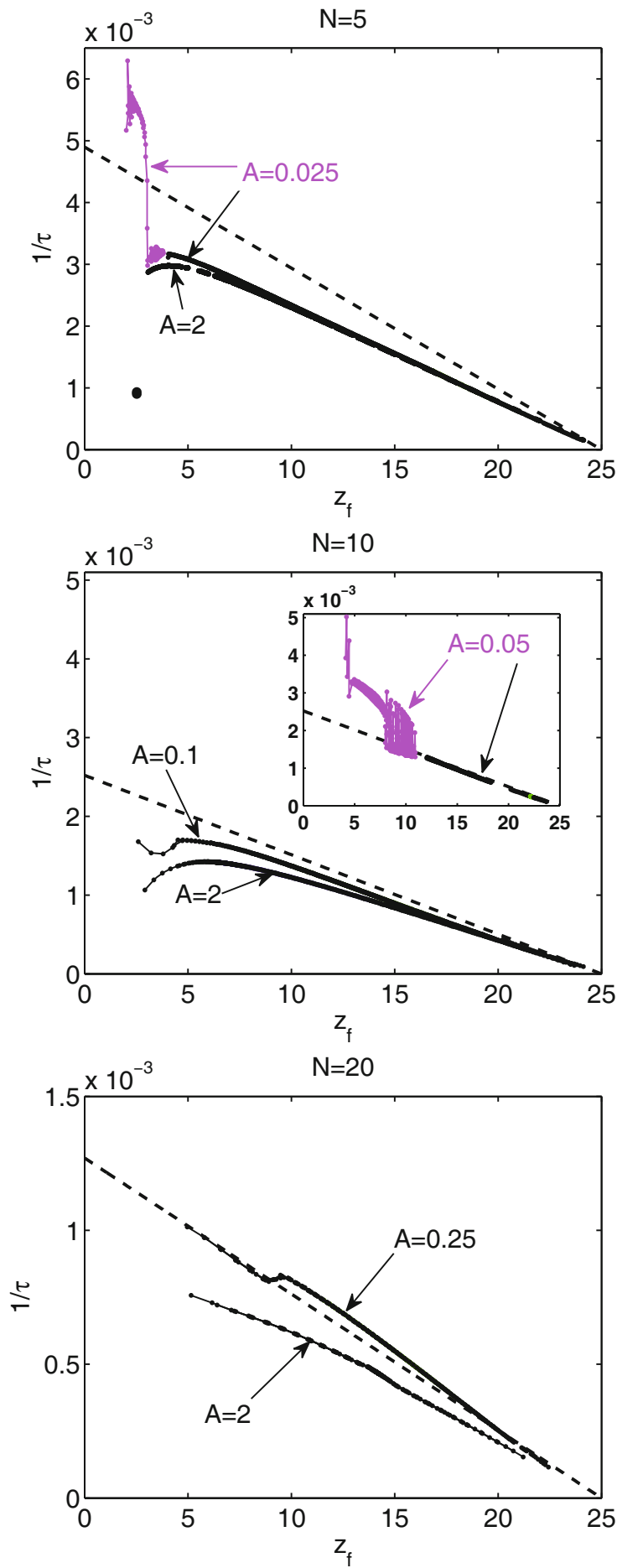

Fig. 15. The fiber tumbling frequency $1 / \tau$ versus its distance $z_{f}$ from the wall (solid lines), in comparison to the inverse half-period of a rigid spheroid with the aspect ratio $N$ (Jeffery, dashed line). Top: $N=5$. Middle: $N=10$. Bottom: $N=20$.

For the Poiseuille flow, the shear rate depends on the position $z$ across the channel,

$$
\dot{\gamma}(z)=8(h / 2-z) / h^{2},
$$

where, in our case, the channel width $h=50$.
In fig. 15, we compare our numerical results for the tumbling frequency of flexible fibers, $1 / \tau$, plotted as a function of $z_{f}$ (solid lines), with the Jeffery's linear relation for the half-period $\dot{\gamma}\left(z_{f}\right) / \pi\left(r_{e}+1 / r_{e}\right)$, which follows from eqs. (19)-(20). For a wide range of relatively larger distances from the wall, the linear dependence $1 / \tau\left(z_{f}\right)$ is in a very good agreement with the Jeffery's expression, with an appropriately chosen, effective value of $r_{e}$. As the reference, we plot the dashed line, which corresponds just to $r_{e}=N$. The surprising effect is that $1 / \tau\left(z_{f}\right)$ is quite well approximated assuming that the effective hydrodynamic aspect ratio $r_{e}$ of flexible fibers is just equal to the number of beads $N$,

$$
r_{e}=N
$$

Unexpectedly, a better agreement is observed for longer and more flexible fibers which deform significantly during the tumbling, with the average geometrical aspect ratio much smaller than $N$.

For smaller distances from the wall, it is known from the literature that the Jeffery approximation is not sufficient owing to the hydrodynamic interaction between the fiber and the wall, see fig. 5 in ref. [2].

The above discussion of fig. 15 has been performed for the regular mode of the dynamics (black online). The irregular mode (violet purple online) is seen as non-smooth, rapidly fluctuating lines, in agreement with the discussion from the previous section.

\section{Conclusions}

In this work, we have considered dynamics of fibers, which are immersed in a low-Reynolds-number Poiseuille flow between two parallel planar solid walls at $z=0$ and $z=h$, and are initially aligned with the flow. Our key finding is that fibers with a different length (i.e. a different number of segments $N$ ) and a different ratio $A$ of the bending stiffness to the flow amplitude, tend to accumulate at a different critical distance $z_{c}$ from the wall. The differences are pronounced. The dependence of $z_{c}$ on $A$ and $N$ has been determined numerically in a wide range of the parameters, based on more than 600 simulation runs.

There exist two different mechanisms of the fiber accumulation. For stiff fibers, hydrodynamic interaction with the close wall prevents them from drifting out of the channel. Therefore, in this case $z_{c}$ is a bit more than half of the fiber length $N$, but still less than $N$. This mechanism (the first type of the accumulation) has been confirmed by the simulations performed for the same fiber and the same Poiseuille flow inside the channel range $0<z<h$, but in the absence of walls. Without walls, stiff fibers migrate away from the channel range $0<z<h$, whatever the initial position is. In contrast, flexible fibers tend to accumulate at larger distances $z_{c}>N$, with similar values in the presence and in the absence of the walls. The mechanism of the second accumulation type is an interplay of the flow curvature, the fiber length and the fiber bending stiffness. 


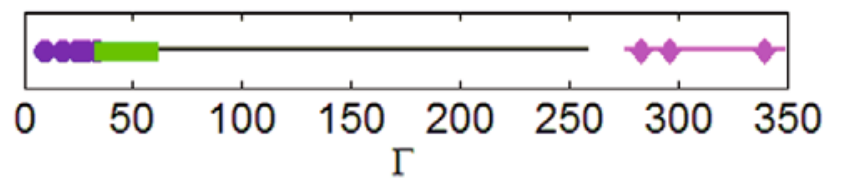

Fig. 16. Values of the shear-to-bending parameter $\Gamma$ for $N=$ 10. Symbols: accumulation positions $z_{c}$ of different types. Solid lines: flipping positions $z_{f}$ of regular and irregular modes.

The comparison with the unbounded flow is in this paper used as the criterion to discriminate between two different types of the fiber accumulation. The additional difference between these two types is that the first one has a smaller fiber curvature and a smaller fractional compression at the accumulation position than the second one.

A third accumulation type has been also detected. Its basic feature is the irregularity of fiber shapes (including large fluctuations of their curvature and fractional compression) at time-dependent accumulation positions. In addition, for the third accumulation type, the fiber curvature and its fractional compression are much larger than for the other accumulation types.

Following the previous literature related to the fiber transitions to higher modes $[9-12,30]$, for $N=10$ we introduced a shear-to-bending parameter $\Gamma=\left(h / 2-z_{f}\right) / A$. (Similar analysis has been also performed for other values of the fiber length $N$.) First, we evaluated values of $\Gamma$ at the accumulation positions $z_{f}=z_{c}$, and found that they are the smallest for the first accumulation type, larger for the second one, and the largest for the third one.

Next, we determined values of $\Gamma$ for all the evaluated flipping positions $z_{f}$, and concluded that for the regular mode of the dynamics, they are smaller than for the irregular one. For the regular mode, a correlation between smaller values of $\Gamma$ and the C-shaped fibers, and between larger values of $\Gamma$ and the S-shaped fibers has been observed for some cases. This problem will be studied in details elsewhere.

In fig. 16, the resulting values of $\Gamma$ are shown. Those corresponding to the accumulation positions are marked by symbols, which for each accumulation type are different, with the shapes and colors consistent with figs. 4, 7 and 8. There exists a sharp threshold $\Gamma_{0}=33.6-36.4$ for activation of the second accumulation type, and a large gap between values of $\Gamma$ corresponding to the second and third types. The values of $\Gamma$ for the regular and irregular modes of the dynamics at the flipping positions $z_{f}$ are indicated by solid lines (black and violet purple online, respectively), with the transition threshold at $\Gamma_{1}=259-275$.

The results presented in this work can be used to sort non-Brownian flexible microfibers, depending on their length and bending stiffness. To this goal, additional measurement of their bending stiffness $A$ is necessary. The analysis presented here indicates that neither the shape evolution nor the tumbling time is sufficient to determine specific value of $A$.

Time and length scales of a fiber migration are relatively large. For example, in a microchannel of width $h=250 \mu \mathrm{m}$, with the maximal Poiseuille flow velocity $v_{m}=1 \mathrm{~mm} / \mathrm{s}$, a fiber of thickness $5 \mu \mathrm{m}$ and length $100 \mu \mathrm{m}$, initially located at the distance $h / 4$ from the wall, typically approaches a distance close to $z_{c}$ after $60-300$ seconds, translating by $50-200 \mathrm{~mm}$.

We thank Professor Jerzy Bławzdziewicz for insightful discussions. This work was supported in part by the Polish Ministry of Science under grant 2011/01/B/ST3/05691.

Open Access This is an open access article distributed under the terms of the Creative Commons Attribution License (http://creativecommons.org/licenses/by/3.0), which permits unrestricted use, distribution, and reproduction in any medium, provided the original work is properly cited.

\section{References}

1. S. Yamamoto, T. Matsuoka, J. Chem. Phys. 198, 644 (1993).

2. M. Zurita-Gotor, J. Bławzdziewicz, E. Wajnryb, J. Rheol. 51, 71 (2006).

3. S.B. Lindstrom, T. Uesaka, Phys. Fluids 19, 113307 (2007).

4. O.B. Usta, J.E. Butler, A.J.C. Ladd, Phys. Rev. Lett. 98, 098301 (2007).

5. R.G. Winkler, J. Chem. Phys. 133, 164905 (2010).

6. C.-C. Huang, R.G. Winkler, G. Sutmannand, G. Gompper, Macromolecules 43, 10107 (2010).

7. A.J.C. Ladd, R. Kekre, J.E. Butler, Phys. Rev. E 82, 050803 (2010).

8. C.-C. Huang, G. Sutmann, G. Gompper, R.G. Winkler, EPL 93, 54004 (2011).

9. L.E. Becker, M.J. Shelley, Phys. Rev. Lett. 87, 198301 (2001).

10. Y.-N. Young, M.J. Shelley, Phys. Rev. Lett. 99, 058303 (2007).

11. E. Wandersman, N. Quennouz, M. Fermigier, A. Lindner, O. du Roure, Soft Matter 6, 5715 (2010).

12. V. Kantsler, R.E. Goldstein, Phys. Rev. Lett. 108, 038103 (2012).

13. L.C. Nitsche, E.J. Hinch, J. Fluid Mech. 332, 1 (1997).

14. R.L. Schiek, E.S. Shaqfeh, J. Fluid Mech. 332, 23 (1997).

15. O.B. Usta, J.E. Butler, A.J.C. Ladd, Phys. Fluids 18, 031703 (2006).

16. R. Chelakkot, R.G. Winkler, G. Gompper, EPL 91, 14001 (2010).

17. S. Reddig, H. Stark, J. Chem. Phys. 135, 165101 (2011).

18. A. Farutin, C. Misbah, Phys. Rev. E 84, 011902 (2011).

19. A. Farutin, C. Misbah, Phys. Rev. Lett. 110, 108104 (2013).

20. E. Gauger, H. Stark, Phys. Rev. E 74, 021907 (2006).

21. A.-K. Tornberg, M.J. Shelley, J. Comput. Phys. 196, 8 (2004).

22. K. Sadlej, E. Wajnryb, M.L. Ekiel-Jeżewska, D. Lamparska, T.A. Kowalewski, Int. J. Heat Fluid Fl. 31, 996 (2010).

23. G. Segré, A. Silberberg, Nature 189, 209 (1961). 
24. O.B. Usta, A.J.C. Ladd, J.E. Butler, J. Chem. Phys. 122, 094902 (2005).

25. R.G. Winkler, Phys. Rev. Lett. 97, 128301 (2006).

26. L. Cannavacciuolo, R.G. Winkler, G. Gompper, EPL 83, 34007 (2008).

27. R. Kekre, J.E. Butler, A.J.C. Ladd, Phys. Rev. E 82, $011802(2010)$

28. X. Xuan, J. Zhu, C. Church, Microfluidics Nanofluidics 9 , $1(2010)$.

29. Y. Kim, J. Yoo, Opt. Lasers Engin. 50, 87 (2012).

30. P.G. de Gennes, J. Chem. Phys. 60, 5030 (1974).

31. B. Cichocki, B.U. Felderhof, K. Hinsen, E. Wajnryb, J. Bławzdziewicz, J. Chem. Phys. 100, 3780 (1994).

32. B. Cichocki, M.L. Ekiel-Jeżewska, E. Wajnryb, J. Chem. Phys. 111, 3265 (1999).

33. S. Kim, S.J. Karrila, Microhydrodynamics. Principles and Selected Applications (Dover Publications, Mineola, 2005).
34. J. Happel, H. Brenner, Low Reynolds Number Hydrodynamics (Nordhoff International Publishing, Leyden, 1973).

35. J.K. Dhont, W. Briels, Rod-like Brownian Particles in Shear Flow (WILEY-VCH Verlag, Berlin, 1988).

36. B.U. Felderhof, Physica A 151, 1 (1988).

37. M.L. Ekiel-Jeżewska, E. Wajnryb, in Theoretical Methods for Micro Scale Viscous Flows, edited by F. Feuillebois, A. Sellier (Transworld Research Network, Kerala, 2009).

38. B. Cichocki, R. Jones, R. Kutteh, E. Wajnryb, J. Chem. Phys. 112, 2548 (2000).

39. S. Bhattacharya, J. Bławzdziewicz, E. Wajnryb, Physica A 356, 294 (2005).

40. A.M. Słowicka, M.L. Ekiel-Jeżewska, K. Sadlej, E. Wajnryb, J. Chem. Phys. 136, 044904 (2011).

41. F. Bretherton, J. Fluid Mech. 14, 284 (1962).

42. G. Jeffery, Proc. R. Soc., London 102, 161 (1922). 